\title{
Janice E. Thompson : le mercenariat comme forme socio-historique de coercition privée
}

\section{Elodie Rigaud}

\section{(2) OpenEdition}

\section{Journals}

Édition électronique

URL : http://journals.openedition.org/conflits/985

DOI : $10.4000 /$ conflits.985

ISSN : $1777-5345$

Éditeur :

CCLS - Centre d'études sur les conflits lilberté et sécurité, L'Harmattan

Édition imprimée

Date de publication : 1 décembre 2003

Pagination : 139-154

ISBN : 2-7475-6065-1

ISSN : 1157-996X

\section{Référence électronique}

Elodie Rigaud, « Janice E. Thompson : le mercenariat comme forme socio-historique de coercition privée », Cultures \& Conflits [En ligne], 52 | hiver 2003, mis en ligne le 03 juillet 2004, consulté le 30 mars 2021. URL : http://journals.openedition.org/conflits/985 ; DOI : https://doi.org/10.4000/conflits. 985

Ce document a été généré automatiquement le 30 mars 2021. 


\title{
Janice E. Thompson : le mercenariat comme forme socio-historique de coercition privée
}

\author{
Elodie Rigaud
}

Selon les études historiques, la première forme de mercenariat est apparue dès l'Antiquité grecque, mais c'est à la Renaissance, entre le XIVème et XVIème siècle, que le phénomène mercenaire a pris une réelle ampleur. En effet, à cette époque en Italie, des condottieri - de condotta, nom des contrats que ces compagnies de mercenaires signaient - étaient employés par les cités italiennes pour se défendre. Remédiant au déclin de l'organisation militaire féodale, les mercenaires constituaient alors de véritables armées professionnelles permanentes. De nos jours, il est souvent fait état d'un retour des mercenaires que l'on inscrit généralement sous l'étiquette équivoque de «nouveaux mercenaires». Ce terme de mercenaires semble réducteur lorsque l'on observe les diverses réalités que le phénomène peut englober et l'hétérogénéité du mercenariat au cours des siècles.

Cette hétérogénéité est non seulement le fait des différents types d'organisation de ces entreprises mercenaires et des services qu'elles proposent, mais également fonction de leur degré de légitimité sur la scène internationale. Le mercenariat, si l'on admet l'utilisation de ce terme générique, fluctue selon l'époque entre un statut d'activité autorisée et non-autorisée par les Etats. C'est cette variabilité dans la gestion des capacités non-étatiques de coercition que Janice Thompson analyse dans son ouvrage Mercenaries, Pirates and Sovereigns, State-building and Extraterritorial Violence in Early Modern Europe ${ }^{1}$. Cet ouvrage présente une réflexion théorique essentielle à la compréhension du phénomène mercenaire car il propose une approche novatrice du lien fonctionnel, ou d'interaction, entre coercition et construction de l'Etat. Cet article ne s'intéressera pas exclusivement à l'analyse des mercenaires proposée par Thompson, mais traitera plus généralement de son approche historique de la coercition externe privée. Pour analyser le phénomène mercenaire, il est en effet nécessaire de le replacer dans le contexte plus large des pratiques de violence privée. Ainsi, l'ouvrage de Janice Thompson approchant de manière globale l'histoire des formes de coercition 
privée offre un cadre utile à l'étude des nouvelles formes de mercenariat émergeant depuis les années 1960.

L'organisation socio-historique de la coercition externe privée au travers de sa relation avec la construction de l'Etat

La construction de l'Etat-nation à l'époque moderne et ses conséquences sur le système international ont donné lieu à une littérature abondante dans les différentes disciplines de sciences sociales. En effet, comme l'a enseigné l'Ecole des Annales et Fernand Braudel, cet objet d'étude se prête tout particulièrement à une réflexion transdisciplinaire qui mobilise les cadres d'analyse de l'histoire, du droit, de la sociologie et des sciences politiques. Les recherches de Charles Tilly, s'appuyant sur une méthodologie de sociologie historique, en sont l'exemple le plus connu. Cet auteur, comme d'autres avant lui, a donné dans son analyse du «state-building " une place centrale à la relation entre autorité politique et violence ${ }^{2}$. En effet, Tilly a mis en parallèle l'organisation de la guerre et le développement de l'Etat-nation comme le résume sa célèbre équation « la guerre fait l'Etat et l'Etat fait la guerre ».

C'est cette même problématique, au travers d'une démarche transdisciplinaire affirmée, que Janice Thompson reprend. Professeur de science politique, elle a cherché au travers de cet ouvrage à affiner les théories de la construction de l'Etat en remettant en question l'idée du monopole de la violence comme attribut essentiel et invariable de l'Etat. Elle refuse de considérer l'organisation actuelle de la violence comme une donnée absolue, elle n'est "ni éternelle ni naturelle ${ }^{3}$. De cette conscience de la relativité naît une interrogation fondamentale: pourquoi et comment une configuration s'impose-t-elle à une autre?

Janice Thompson, s'intéressant plus particulièrement aux moyens de violence externe, défend la thèse suivant laquelle "le 'désarmement' des activités transnationales nonétatiques a marqué la transition de l'hétéronomie à la souveraineté et la transformation des Etats en un système d'Etats nationaux $»^{4}$, transformation mettant en place un nouveau jeu d'interactions internationales fortes entre des Etats qui se sont auparavant imposés comme autorité légitime au niveau national. La souveraineté et l'Etat national se sont donc construits parallèlement à la mise en place d'une nouvelle organisation des ressources mondiales de coercition. Janice Thompson définit un système hétéronome comme un système dans lequel la violence est démocratisée, commercialisée et internationalisée et donc, en somme, disséminée. Selon elle, ce système hétéronome s'est mué progressivement en un système d'Etats théoriquement autonomes et souverains, les Etats s'efforçant désormais d'avoir un contrôle effectif sur les moyens de coercition. Ainsi, à une monopolisation étatique de la violence interne succède celle de la violence externe. Cette évolution s'est accomplie sous l'impulsion du système luimême, système conçu comme la collectivité des dirigeants des Etats européens : «la délégitimation et l'abolition de la violence non-étatique ont été le résultat d'interactions entre les dirigeants des Etats $»^{5}$. Le changement de système ne serait donc pas la réponse à une hypothétique demande provenant de la société.

$\mathrm{Au}$ travers de cette thèse, Janice Thompson touche aux concepts centraux des sciences politiques, en particulier à celui de l'Etat. Elle critique la conception weberienne de l'Etat en soulignant la confusion analytique qui s'opère lorsque l'on considère le contrôle de la violence comme le trait caractéristique de l'Etat. En effet, la violence contrôlée par l'Etat traditionnel n'est pas de même nature que celle contrôlée par l'Etat-nation. Dire que l'Etat est défini par son monopole de la violence est insuffisant 
car l'on ne précise pas si cette violence est interne/domestique ou externe/ internationale. Son analyse concerne également le concept de souveraineté qui est sujet à des définitions diverses selon les théoriciens. Janice Thompson ramène cette souveraineté à une construction normative ; les normes de souveraineté émergeraient des interactions entre les Etats.

Dans le premier chapitre, "The State, Violence and Sovereignty ", Janice Thompson revient ainsi sur les différentes théories relatives à l'Etat, la violence et la souveraineté. Partant des différentes définitions que donnent Max Weber, Charles Tilly et Anthony Giddens de l'Etat, elle démontre que la variabilité des caractéristiques que l'on prête à cette institution n'est en fait que le reflet de l'évolution historique de la nature de l'Etat. Alors que Weber fait correspondre Etat et contrôle de la coercition sans plus de précisions, Tilly y ajoute la notion de territoire et Giddens précise que ce contrôle s'exerce "sur les moyens de violences internes et externes». A cela s'ajoutent des divergences concernant d'une part la nature légitime de ce contrôle, et d'autre part son objet, à savoir l'usage et/ou les moyens de la violence. Alors qu'elle passe rapidement sur ce problème de légitimité en soulignant la nécessité de déterminer la source de légitimité - l'usage de la violence est-il légitime pour la population ou l'Etat ? - l'auteur s'attarde davantage sur la distinction à faire entre l'usage et les moyens de la violence. Cette distinction est très enrichissante pour la réflexion théorique. Elle permet de distinguer des étapes dans l'évolution du contrôle étatique de la violence en croisant plusieurs variables comme celles de l'autorité de décision et de la détention des moyens. Un tableau à plusieurs entrées illustre ainsi parfaitement le fait que «le contrôle de l'Etat sur la violence n'est pas seulement multidimensionnel mais aussi hautement variable $»^{6}$. On comprend alors qu'au XIXème siècle, les équations possibles diminuent parce que l'Etat possède à la fois l'autorité de décision et ses propres moyens de violence.

En ce qui concerne la souveraineté, Janice Thompson reproche aux théories réalistes de la concevoir comme un attribut définitif de l'Etat, ce qui rend impossible l'analyse du changement. Elle ne semble pas non plus adhérer aux thèses interdépendantistes qui, en opposition au réalisme, considèrent que la souveraineté de l'Etat est érodée par le jeu des d'interdépendances. La non-adhésion de Janice Thompson aux théories interdépendantistes est d'ailleurs confirmée dans un article postérieur à cet ouvrage dans lequel elle dénonce leur conception an-historique de la souveraineté et centrée sur le contrôle aux dépens de l'autoritép. Selon elle, la souveraineté d'un Etat ne se résume pas seulement à sa capacité effective à contrôler son champ d'action, mais elle dépend également de sa capacité à décider quelles activités entrent ou sortent de celuici. Aux paradigmes réaliste et interdépendantiste, elle préfère l'approche des théoriciens critiques, tels John Ruggie, Richard Ashley et R.B.J. Walker. Ces derniers considèrent la nature de la souveraineté, de la même manière que tous les types de frontières, comme socialement construite. Janice Thompson inscrit donc sa démarche dans le cadre du courant critique tout en essayant par sa méthodologie comparative et historique de l'étoffer par une illustration empirique qui, selon elle, fait souvent défaut aux travaux des auteurs critiques. De même, elle suit le paradigme institutionaliste en analysant la souveraineté comme une institution construite autour des deux dimensions «constitutive» et "fonctionnelle» de la souveraineté. D'une part, la dimension constitutive, qui consiste en la monopolisation de la violence par l'Etat sur un territoire donné, permet à l'Etat de se poser en acteur principal sur la scène internationale et de déterminer la frontière entre les politiques interne et externe. 
D'autre part, la dimension fonctionnelle, qui concerne l'espace politique sur lequel l'autorité de l'Etat s'étend, établit les limites entre l'économique et le politique, ainsi qu'entre les champs respectifs de l'Etat et des acteurs non-étatiques. La souveraineté dépendrait alors de l'évolution de ces deux dimensions complémentaires. Elle changerait de nature selon les attentes externes, c'est-à-dire celles du système international, et selon l'étendue du champ d'autorité revendiqué par l'Etat.

Janice Thompson propose trois hypothèses pour expliquer le changement dans l'organisation de la violence: ce changement est le résultat des pratiques de la collectivité des dirigeants des Etats, même si les formes de violence non-étatique et leur contrôle dépendent du système politique interne; il n'est pas intentionnel; les capacités physiques de puissance n'en sont pas un facteur décisif. Pour valider sa thèse, Janice Thompson analyse le changement sur le long terme suivant ainsi, par souci méthodologique, les théoriciens du système-monde et de la longue durée que sont Fernand Braudel, Anthony Giddens, Immanuel Wallerstein et Michael Mann. Comme eux, elle accorde une grande importance à l'histoire et à la dimension temporelle tout en refusant le biais évolutionniste puisque dès les premières pages elle s'oppose aux thèses téléologiques, fonctionnalistes et déterministes. Elle prend comme méthode d'analyse celle de la " narration historique ", expression de Michael Mann, méthode qui consiste en un raisonnement inductif s'appuyant sur une approche comparative et historique. Ainsi, elle pose comme variable dépendante l'élimination de la violence non-étatique et, après avoir identifié les différentes formes de violence non-étatiques et comparé leurs processus de délégitimation respectifs, elle peut établir les variables indépendantes faisant le lien entre causes et résultat. Cet ouvrage, s'inspirant de la théorie des relations internationales, du droit international, de la sociologie et de l'histoire, ne propose donc pas de nouvelles données. Cependant, il apporte un nouvel éclairage à l'étude de la violence en analysant les pratiques internationales de violence non-étatique, parmi lesquelles le mercenariat constitue un des exemples les plus représentatifs.

Les pratiques de coercition privée et leur gestion à travers l'histoire : genèse et élimination de la violence non-étatique externe

L'organisation du contenu de l'ouvrage reflète la démarche analytique de l'auteur : elle part de l'origine des formes principales de violence non-étatique pour arriver aux causes de leur élimination. En reprenant les différentes formes de violence nonétatique, on peut se rendre compte que cette violence est démocratisée, commercialisée et internationalisée par l'action des dirigeants européens eux-mêmes, ces derniers cherchant par ce biais à échapper aux contraintes du système féodal. En effet, les monarques ne pouvaient forcer leurs vassaux à servir militairement à l'extérieur du pays pour une durée indéterminée puisque le service d'ost, service militaire, était limité dans le temps et ne pouvait être que défensif. Par ailleurs, dès la fin du MoyenAge, il devint possible pour chaque fief de se racheter de ce service moyennant le versement d'une somme d'argent. Grâce à cet impôt, les monarques entretenaient des mercenaires et institutionnalisaient ainsi la violence privée à des fins de politique étrangère. La distinction que fait l'auteur entre les formes autorisées de violence nonétatiques et celles non-autorisées amène à considérer ces dernières comme les conséquences non intentionnelles de cette tendance à autoriser la violence nonétatique. Selon Janice Thompson, au XIXème siècle, se produit une transition marquée par l'abolition de la violence non-étatique autorisée sous l'impulsion d'interactions interétatiques favorisant la mise en place de normes d'interdiction de ces pratiques. De 
la délégitimation normative de ces pratiques découlent ensuite les efforts pour éliminer les formes traditionnelles de violence non-étatique et non-autorisée.

Janice Thompson, dans son deuxième chapitre intitulé «Nonstate Violence Unleashed », recense trois formes de violence non-étatique autorisée progressivement apparues à partir du XIIIème siècle : les corsaires, les compagnies marchandes et les mercenaires. Contrairement aux pirates qui poursuivaient leurs propres intérêts, les corsaires agissaient théoriquement sous l'autorité d'un Etat qui était responsable de leurs actes. Cette pratique prenait une ampleur considérable pendant les conflits et les guerres interétatiques. Elle représentait un substitut et même une base pour la puissance navale de l'Etat. Les compagnies commerciales, créées au XVIème siècle en Europe, représentent la forme la plus complexe de violence non-étatique autorisée. Ces compagnies étaient affrétées par l'Etat pour faire du commerce sur de longues distances ou pour établir des colonies. Les plus importantes étaient les deux Compagnies hollandaise et britannique des Indes Orientales et la Compagnie de la Baie d'Hudson. Leur organisation différait d'une compagnie à l'autre et leur degré de privatisation par rapport à l'Etat. Par conséquent, leurs objectifs (pouvoir/profit) variaient selon que les pays d'origine étaient plus ou moins centralisés. Ces compagnies recevaient des pouvoirs extraordinaires qui leur conféraient une quasi-souverainetéé. Janice Thompson résume la relation ambiguë, allant jusqu'à la collusion d'intérêts, entre l'Etat et ces compagnies par la phrase suivante: "les compagnies marchandes étaient des institutions créées par l'Etat qui utilisaient la violence dans la poursuite d'un gain économique et de pouvoir politique à la fois pour l'Etat et des acteurs non-étatiques " ${ }^{8}$. Quant aux mercenaires, comme nous l'avons vu précédemment, ils constituent l'exemple type de stratégie étatique pour échapper aux lourdeurs du système féodal concernant les obligations militaires. Au XVIIIème siècle, la pratique du mercenariat était telle que pratiquement tous les Etats employaient et fournissaient des troupes mercenaires, les armées devenant ainsi de véritables forces multinationales. Les mercenaires venaient alors majoritairement des Etats allemands, des Pays-Bas, d'Angleterre, de Venise et de Suisse, ces pays "fournisseurs" de mercenaires étant eux-mêmes "employeurs » d'étrangers dans leurs armées. Ces échanges d'hommes entre les pays étaient un enjeu stratégique très important. Ainsi, en 1516, la Suisse signe-t-elle un accord avec la France promettant de ne jamais fournir de mercenaires aux pays ennemis de la France. Les mercenaires étaient également utilisés dans la marine, surtout dans la marine anglaise dont près de la moitié de l'effectif était constitué d'étrangers. Les Etats européens ont donc exploité les capacités de coercition de ces acteurs non-étatiques corsaires, compagnies commerciales et mercenaires. Mais, progressivement, les problèmes «inattendus et ingérables » produits par ces pratiques ont surpassé les bénéfices que pouvaient en tirer les Etats. Plus encore, il devint impossible pour l'Etat d'encourager la violence non-étatique sans en assumer une responsabilité.

Ces conséquences non-intentionnelles, comme le titre le troisième chapitre, sont propres à chacune des formes de violence non-étatique autorisée. La pratique des corsaires a généré la piraterie organisée. Alors que les corsaires de la Méditerranée pouvaient déjà être assimilés à des pirates, la piraterie au XVIème siècle acquiert une nature politique. Les pirates, comme les Boucaniers ou le 'commonwealth' des pirates de Madagascar, sont alors des « communautés ou des quasi-Etats basés sur le principe de la démocratisation des pouvoirs politiques et de la violence " ${ }^{9}$. Les compagnies marchandes, quant à elles, ont utilisé leurs capacités militaires contre les autres compagnies, indépendamment des relations que pouvaient entretenir leurs Etats d'origine 
respectifs, mais aussi directement à l'encontre des Etats européens. De même, l'utilisation de mercenaires a engendré le risque d'entraîner les Etats les employant dans des guerres contre d'autres Etats. Ce risque est apparu avec l'émergence des pratiques et des principes associés à la neutralité qui rendent responsable l'Etat pour les actions internationales d'individus placés sous sa juridiction. Le mercenaire n'était plus alors un simple acteur du marché, il devenait un acteur politique et, en tant que tel, ses actes pouvaient être imputés aux Etats. Ces conséquences sont en fait la marque d'un manque de contrôle effectif de l'Etat sur ces entreprises de violence non-étatiques. Or, ce manque est né, paradoxalement, d'une stratégie volontaire qui consistait à "maximiser l'effectivité des acteurs non-étatiques» en "minimisant les contraintes sur leurs activités et profits $»^{10}$. En effet, cette stratégie avait initialement eu l'avantage de réduire au minimum toute imputabilité de l'Etat concernant les actions de ces entreprises. En somme, les conséquences involontaires de ces pratiques consistaient dans leur ensemble à «donner aux individus le pouvoir d'agir indépendamment de leur Etat d'origine ", de potentiellement agir contre ses intérêts et donc de le défier.

$\mathrm{Au}$ quatrième chapitre, "Delegitimating State-Authorized Nonstate Violence ", Janice Thompson montre qu'éliminer ces problèmes nés de pratiques légitimées depuis plusieurs siècles n'était pas une chose évidente. Chaque pratique a dû subir un processus de délégitimation propre. Un accord international formel a ainsi aboli l'utilisation des corsaires. En effet, le traité de Paris en 1856 a amorcé une uniformisation internationale du droit maritime excluant progressivement les activités des corsaires. Les compagnies marchandes, quant à elles, ont été éliminées au travers de différents processus sans jamais être formellement interdites. La plupart a disparu suite à des faillites et certaines ont progressivement perdu leur contrat d'affrètement, comme la Compagnie britannique des Indes orientales qui représentait un enjeu de lutte entre la monarchie et le parlement. Enfin, alors que la délégitimation des corsaires a été le fruit d'une évolution dans le droit international, celle des mercenaires est due à un renforcement du droit interne (Communal law). Les pratiques mercenaires ont progressivement perdu leur légitimité à la suite de la promulgation par les EtatsUnis de lois de neutralité en 1794 et 1818 qui considèrent comme délit le fait qu'un citoyen américain prépare ou prenne part à un conflit à l'étranger. Ces lois qui codifiaient, pour la première fois de façon permanente, les droits et devoirs des Etats neutres, ont entraîné au XIXème siècle une vague de législations anti-mercenaires internes. Ainsi, le droit interne, qui établissait des normes plus strictes à l'intérieur du pays, a eu des répercussions extérieures, le jeu des interactions internationales contraignant les Etats à généraliser ces nouvelles normes : «la politique de neutralité américaine est devenue universelle parce qu'elle créait de nouvelles attentes concernant le comportement des Etats neutres $\aleph^{11}$. Mais en plus de répondre à ces attentes, les régulations anti-mercenaires reflètent les nouvelles relations entre l'Etat et la population, le premier cherchant à mieux contrôler les secondes à l'intérieur de ses frontières. Les formes de violence non-étatique autorisée ont donc été «éliminées d'une manière ad hoc et au coup par coup $\aleph^{12}$, mais toujours sous l'impulsion des dirigeants des Etats européens.

Les formes de violence non-étatique non-autorisée sont difficilement identifiables car la frontière séparant ce qui est autorisé par l'Etat de ce qui ne l'est pas est loin d'être fixe et clairement définie. Deux pratiques non-autorisées sont abordées au sixième chapitre, "Suppressing Unauthorized Nonstate Violence », de cet ouvrage : la piraterie et la flibusterie. La piraterie est peu à peu passée du statut de pratique non-autorisée, 
mais hors de la responsabilité des Etats et potentiellement exploitable, à celui d'activité criminelle que l'Etat devait éliminer. Ainsi, « la campagne contre la piraterie a été précédée par un changement dans l'attitude de l'Etat $~^{13}$. Cette élimination n'a pu être réellement effective qu'une fois dépassé l'obstacle de l'identification des responsabilités. Tout le problème consistait à savoir qui était souverain en mer et donc responsable des actions des pirates. La question n'a finalement pas été tranchée et les normes d'interdiction et de poursuite des pirates ont été générées par le seul droit interne (Communal law). La flibusterie, quant à elle, était une nouvelle forme de violence extraterritoriale apparue après l'établissement d'un gouvernement républicain aux Etats-Unis. Cette pratique, exclusivement limitée au continent américain, consistait en des expéditions militaires non-étatiques contre des territoires voisins. Trois types d'expéditions sont étudiés par l'auteur: d'abord, celles mises en place par des initiatives privées de nationaux étrangers ou de citoyens américains, puis celles résultant des efforts malencontreux des Mexicains pour attirer les résidents américains, et enfin celles conduites par des agents du gouvernement américain. Ces trois types de flibusterie ont pour point commun leur nature politique complexe, produit de la faiblesse de l'Etat fédéral face aux Etats et aux citoyens. La lutte contre cette pratique correspond donc à " un effort de l'Etat à étendre et renforcer son contrôle sur les individus à l'intérieur de sa juridiction territoriale $»^{14}$.

Le monopole de l'Etat sur les formes de coercition internationale n'a, par conséquent, été effectif qu'à partir du XIXème siècle lorsque la violence non-étatique externe, qu'elle soit autorisée ou simplement tolérée, a perdu sa légitimité et a été éliminée. Si l'on reprend le tableau analytique de l'organisation de la violence, la violence externe a été ramenée par ce long processus aux seules variables «allocation par l'Etat » et « détention par l'Etat ».

Perspectives théoriques et réflexions critiques à l'aune du renouvellement et des transformations du mercenariat depuis les années 1960

Cet ouvrage, par sa profusion de détails historiques, a réussi à décrire les processus par lesquels les dirigeants étatiques ont encouragé puis éliminé la violence extraterritoriale non-étatique. Cette analyse remet en cause l'assimilation simplificatrice de l'Etat à son monopole sur la violence. Ce monopole n'est pas intentionnel, il doit être analysé comme le résultat d' "une série d'étapes dans l'évolution de l'autorité et du contrôle de $l^{\prime}$ Etat $»^{15}$. En relativisant ainsi l'idée traditionnelle selon laquelle l'Etat s'efforce volontairement à monopoliser la violence, l'auteur met en relief l'importance de la collectivité des Etats sur le comportement de chacun d'entre eux. Janice Thompson explique cette transition dans l'organisation de la violence non seulement par l'accumulation de ressources propres qui accroît les capacités étatiques de contrôle, mais aussi par un effet «boule de neige " qui étend les domaines de contrôle. La monopolisation de la violence par l'Etat est le résultat d'un «isomorphisme institutionnel » qui implique que «tous les Etats adoptent l'interdiction de la violence nonétatique $»^{16}$. L'expansion européenne puis la montée du gouvernement républicain ont été les conditions de cette transition: «la violence non-étatique était en accord avec la souveraineté de l'Etat, mais elle ne le fut plus avec la souveraineté de l'Etat national $\aleph^{17}$. En insistant sur ces deux conditions, Janice Thompson s'éloigne encore de la thèse réaliste qui conditionne l'importance de la monopolisation de la violence aux seules capacités de puissance. La reconnaissance de la souveraineté par les autres Etats devient ainsi la principale source de pression pesant sur les dirigeants étatiques pour qu'ils modifient leurs normes. On peut rapprocher cette réflexion de la théorie des régimes qui explique 
l'obligation de coopérer par l'établissement d'un ensemble de normes créant un faisceau d'attentes. Répondre à ces attentes devient alors le comportement le moins coûteux. Les institutions, ainsi mises en place, fondent sur le long terme des formes de loyauté. Pourtant, limiter l'explication de l'élimination des formes de violence nonétatique à des impératifs de politique étrangère peut être critiquable car cela met dans l'ombre les luttes internes à l'Etat qui entouraient ces pratiques. Il aurait sûrement été enrichissant pour l'analyse de souligner de façon plus prononcée le manque de cohésion politique à l'intérieur de l'Etat. Cela aurait permis d'identifier les divers acteurs politiques concernés, acteurs qui par leurs interactions plus ou moins conflictuelles ont poussé à la légitimation puis à l'élimination de cette violence.

Janice Thompson conclut son ouvrage en indiquant les implications théoriques de sa recherche. Celles-ci concernent aussi bien la coopération interétatique - les relations interétatiques ont joué un « rôle crucial » dans le façonnement des " relations entre l'Etat et la société $\aleph^{18}$-, que l'étude des pays en développement qui n'ont plus la possibilité de renforcer leur Etat par le biais de la violence non-étatique, comme l'ont fait les Etats occidentaux. En effet, ces derniers ont délégitimé cette violence. De même, la théorie de normes s'en trouve précisée puisque l'analyse de la monopolisation de la violence a démontré que les normes sont les conséquences non-intentionnelles des interactions interétatiques. Alors que Janice Thompson semble déplorer le fait que cette recherche fait émerger plus de questions concernant l'Etat et la souveraineté qu'elle n'apporte de réponses, c'est justement à cet apport de questionnement que l'on reconnait la richesse du travail entrepris puisqu'il fait progresser la recherche en alimentant la réflexion théorique. De plus, l'accent que l'auteur met sur la variabilité des concepts tels que l'Etat et la souveraineté apparaît essentiel pour élargir le champ de la connaissance, mais aussi pour accepter l'existence ou la possibilité de configurations alternatives et donc mieux comprendre le changement.

Par contre, même si Janice Thompson admet que «l'exploitation de la violence nonétatique reste une tentation puissante pour les dirigeants des Etats", elle semble minimiser les pratiques de violence non-étatique qui subsistent au XXème siècle. En effet, selon elle, les cas contemporains de mercenariat, qu'ils constituent des forces permanentes d'armées nationales régulières ou des forces ad hoc, ne représentent que des anomalies puisqu'ils ne sont légitimés que marginalement. Il est vrai que les deux guerres mondiales du XXème siècle ont encore accentué l'illégitimité de l'usage de mercenaires.

Cependant, bien que les mercenaires n'apparaissent plus dans les conflits interétatiques, les conflits infra-étatiques, qui ont moins de visibilité, leur offrent une nouvelle aire d'intervention. Ces interventions, limitées à ce type de conflits, correspondent à une coercition interne mais aussi, d'une manière plus ou moins indirecte, à une coercition externe. En effet, bien qu'elles concernent en premier lieu les affaires internes à un pays donné, elles s'inscrivent dans le cadre, si ce n'est de logiques d'ingérence, tout du moins de politiques étrangères d'acteurs extérieurs au pays. Ainsi, durant les années 1960, la décolonisation en Afrique a été marquée par de nouvelles figures du mercenariat comme le Français Bob Denard, l'Irlandais Mike Hoare, ex-major de l'armée des Indes, ou le Belge Christian Tavernier. Ces mercenaires ont conduit des bandes dans des pays comme le Congo, les Comores, les Seychelles ou l'Angola. Employés pour vaincre les mouvements africains d'indépendance, ces mercenaires de la deuxième moitié du XXème siècle n'ont rien en commun avec les condottieri de la Renaissance et sont effectivement hors des normes du système 
international. Mais qu'en est-il des compagnies militaires privées et des sociétés de sécurité privée lorsqu'on les considère comme des formes contemporaines du mercenariat?

Les premières compagnies militaires privées datent de la fin des années soixante. En 1967, le Colonel David Stirling a fondé WatchGuard International, une compagnie qui employait d'anciens officiers de la Special Air Service britannique (S.A.S.) pour entraîner des militaires à l'étranger. La société sud-africaine Executive Outcomes, qui n'existe plus depuis 1998, avait été fondée sur ces modèles. Aujourd'hui, les sociétés qui entrent dans cette catégorie sont l'américaine Military Professional Resources Inc., la britannique Sandline, les firmes israéliennes Silver Shadow et Levdan, la Compagnie Française d'Assistance Spécialisée (C.O.F.R.A.S.), la société belge International Defence and Security (I.D.A.S.) et Teleservices et Alpha 5 qui sont deux sociétés angolaises. D'autres sociétés, souvent associées aux compagnies militaires mais distinctes d'elles de par leur nature, apparaissent également. Elles fournissent des services très spécialisés ayant une application militaire, mais ne sont pas nécessairement elles-mêmes militaires ou même paramilitaires dans leurs organisations ou leurs méthodes. Ce sont des compagnies de sécurité privée (PSCs) parmi lesquelles les plus connues sont les sociétés britanniques Lifeguard, Group 4 et Securicor, les sociétés américaines Dyncorp et Kroll Associates, ainsi que la Gray Security et la Coin Security d'Afrique du Sud.

Ces dernières années, ces deux types d'activité se sont particulièrement développés pour pratiquement se confondre, les compagnies militaires privées adoptant le statut moins controversé de PSC tandis que les sociétés de sécurité privée offrent des services de double nature. La législation internationale semble manquer de moyens pour réguler ces forces non-gouvernementales de sécurité. En effet, ces sociétés de sécurité privée, lorsqu'elles se limitent à proposer des applications militaires ou paramilitaires à des technologies civiles, échappent à toute poursuite puisqu'elles ne sont, dans ce cas, contraintes que par les clauses d'un contrat commercial. Elles échappent donc à la législation anti-mercenaire que le système des Etats-nations a mis en place. Ainsi, alors que la délégitimation normative décrite par Janice Thompson a rendu hors-la-loi le mercenariat, ces sociétés tombent difficilement sous l'accusation d'illégalité.

Ces sociétés de sécurité privée semblent reprendre toutes les caractéristiques des pratiques autorisées de coercition privée des époques médiévale et moderne étudiées par Janice Thompson. Ces sociétés entretiennent des liens souvent obscurs avec, d'une part les entreprises commerciales, et d'autre part leurs Etats d'origine ou d'accueil. Ces Etats semblent hésiter entre une condamnation pure et simple de ces sociétés en tant qu'activité mercenaire, et un discours plus nuancé qui permettrait à ces sociétés d'exercer leur activité dans un cadre défini par les Etats eux-mêmes. Comme les mercenaires, corsaires et compagnies marchandes du XIIIeme siècle, les sociétés de sécurité privée représentent par leurs capacités de coercition, ou tout du moins de sécurité, un possible nouveau mode de gestion de l'insécurité. Dan Nelson, expert de la défense au George $\mathrm{C}$. Marshall Center for Security Studies présente ce phénomène comme " une politique étrangère par personnes interposées. Des entités constituées sont utilisées pour accomplir les tâches que le gouvernement, pour des raisons budgétaires ou par délicatesse politique, ne peut prendre en charge $»^{19}$.

Il serait donc utile d'étudier ce phénomène au travers de la grille d'analyse proposée par Janice Thompson: N'y a-t-il pas un retour à une allocation mixte de la violence par l'Etat et par des acteurs non-étatiques de la violence - associée au transfert des 
moyens de la violence à des acteurs non-étatiques? Et, dans ce cas, comment les Etats sont-ils prêts à faire face aux " problèmes inattendus et ingérables » que l'usage de ces sociétés de sécurité privé impliquerait? Enfin, si les moyens de la contrainte deviennent non-gouvernementaux, qu'en est-il de l'autorité sur l'exercice de ces moyens ? Est-elle étatique, privée ou mixte? En somme, sans pour autant en conclure que l'on assiste à une réorganisation hétéronome de la violence, l'emploi de ces sociétés est, peut-être, le signe d'une nouvelle variation des domaines déclarés d'autorité et de responsabilité de la souveraineté.

Bibliographie du même auteur

Articles

THOMPSON Janice E., "States Practices, International Norms, and the Decline of Mercenarism », International Studies Quarterly, 34, mars 1990, pp. 23-47.

"Sovereignty and the Institutional Isomorphism of States", rapport présenté à la réunion annuelle de l'American Political Science Association, Washington, D.C., du 29 août au 1er septembre 1991.

« Norms in International Relations: a Conceptual Analysis ", International Journal of Group Tensions, 23, printemps 1993, pp. 67-83.

"State Sovereignty in International Relations : Bridging the Gap Between Theory and Empirical Research », International Studies Quarterly, 39, 1995, pp. 213-233.

Ouvrages

THOMPSON Janice E., KRASNER Stephen D., « Global Transactions and the Consolidation of Sovereignty ", in CZEMPIEL Ernst-Otto, ROSENAU James N. (eds.), Global Changes and Theoretical Challenges: Approaches to World Politics for the 1990s, Lexington, Lexington Books, 1989.

THOMPSON Janice E., « Explaining the Regulation of Transnational Practices : a StateBuilding Approach ", in ROSENAU James N., CZEMPIEL Ernst-Otto (eds.), Governance without Government, Cambridge, Cambridge University Press, 1992.

\section{NOTES}

1. . Thompson Janice E., Mercenaries, Pirates and Sovereigns : State-building and Extraterritorial Violence in Early Modern Europe, Princeton University Press, 1994.

2. . Voir notamment : Tilly Charles, Contrainte et capital dans la formation de l'Europe, 990-1990, Paris, Aubier, 1992.

3. . Thompson Janice E., op. cit., p. 3.

4. . Ibid, p. 4.

5. . Ibid, p. 4.

6. . Ibid, p. 9.

7. . Thompson J. E., « State Sovereignty in International Relations : Bridging the Gap Between Theory and Empirical Research », International Studies Quarterly, 39, 1995, pp. 213-233. 
8. . Thompson Janice E., op. cit., 1994, p. 41.

9. . Ibid, p. 46.

10. . Ibid, p. 43.

11. . Ibid, p. 84.

12. . Ibid, p. 105.

13. . Ibid, p. 108.

14. . Ibid, p. 143.

15. . Ibid, p. 145

16. . Ibid, p. 147

17. . Ibid, p. 148.

18. . Ibid, p. 150

19. . Cité par Silverstein Ken, Private Warriors, Londres, Verso, 2000, p. 145.

INDEX

Thèmes : Executive Outcomes, MPRI

Index chronologique : Moyen Age, 18ème siècle, 19e siècle

Mots-clés : privatisation de la sécurité, Relations Internationales, sociologie historique 